\title{
Short-term outcomes of preoperative chemoradiotherapy with S-1 for locally advanced rectal cancer
}

\author{
MASATSUGU HIRAKI ${ }^{1}$, TOSHIYA TANAKA ${ }^{1}$, YOSHIKI HIRAKI ${ }^{2}$, TETSUO WATANABE ${ }^{2}$, \\ HIROFUMI SATO $^{1}$, HITOSHI AIBE ${ }^{2}$ and KENJI KITAHARA ${ }^{1}$ \\ Departments of ${ }^{1}$ Surgery and ${ }^{2}$ Radiology, Saga Medical Center Koseikan, Kasemachi, Saga 840-8571, Japan
}

Received May 17, 2020; Accepted September 9, 2020

DOI: $10.3892 / \mathrm{mco} .2020 .2166$

\begin{abstract}
The purpose of the present study was to evaluate the short-term results of preoperative chemoradiation therapy with S-1 for locally advanced rectal cancer. A total of 32 patients with advanced rectal cancer who had been treated with preoperative chemoradiotherapy with $\mathrm{S}-1$ and underwent surgical resection between May 2012 and December 2019 were analyzed. Advanced rectal cancer of clinical stage II and III was diagnosed in $13(41 \%)$ and 19 (59\%) patients, respectively. Therapeutic toxicities of anemia (24 patients; 75\%), anal pain (22 patients; 69\%) and skin and subcutaneous tissue disorders (19 patients; 59\%) were frequently observed in all grades. Grade $\geq 3$ leukopenia, anemia, neutrophil count reduction, platelet count reduction and diarrhea were identified in $2(6 \%)$, $1(3 \%), 1(3 \%), 1(3 \%)$ and $1(3 \%)$ patients, respectively. A total of 29 patients $(91 \%)$ completed this therapy without any change to the protocol or dosage. R0 resection was performed in $100 \%$ of the patients, and no postoperative mortality was observed. Pathological complete response was observed in 9 cases $(28.1 \%)$. This therapy can be considered for cases of locally advanced rectal cancer due to its acceptable toxicity and relatively high antitumor effect.
\end{abstract}

\section{Introduction}

In recent years, multimodal treatment including initial preoperative chemoradiotherapy (CRT) followed by total mesorectal excision has become the preferred treatment for advanced rectal cancer, and this strategy is widely accepted as a standard treatment in western countries (1). Randomized clinical trials have showed that fluorouracil-based preoperative CRT reduces local recurrence compared with preoperative radiation without chemotherapy or postoperative CRT (2-4). Conventional

Correspondence to: Dr Masatsugu Hiraki, Department of Surgery, Saga Medical Center Koseikan, 400 Nakabaru, Kasemachi, Saga 840-8571, Japan

E-mail: masatsuguhiraki@hotmail.com

Key words: preoperative chemoradiation therapy, chemoradiation therapy, neoadjuvant therapy, rectal cancer, S-1 intravenous 5-fluorouracil (5-FU) or capecitabine have been used as radiosensitizing agents in CRT in recent years (1). However, further improvements in this therapy are needed in order to achieve better outcomes of cancer treatment and a better quality of life for patients. Therefore, different chemotherapeutic regents and regimens as radiosensitizers have been recently attempted for CRT (5).

$\mathrm{S}-1$, a biochemical modulator of 5-FU, is an oral dihydropyrimidine dehydrogenase inhibitory fluoropyrimidine containing tegafur, gimeracil (5-choro-2,4-dihydroxypyridine) and potassium oxonate in a molar ratio of 1:0.4:1 (6). Tegafur is a prodrug of 5-FU, and gimeracil is a competitive inhibitor of dihydropyrimidine dehydrogenase that degrades 5-FU (6). S-1 contains not only tegafur as a radiosensitizer but also gimeracil, which enhances the efficacy of the radiotherapy (7-9). However, few studies regarding preoperative CRT with S-1 for rectal cancer have been conducted thus far (10-12).

The present study therefore investigated the short-term outcomes of neoadjuvant CRT with S-1 for locally advanced rectal cancer.

\section{Patients and methods}

Eligibility criteria. The inclusion criteria were as follows: i) Histologically confirmed primary adenocarcinoma of the rectum and the tumor was mainly or partially located in the lower rectum; ii) tumor stage of cT3-4 with any $\mathrm{cN}$, including clinically metastasis to lateral pelvic lymph nodes, and $\mathrm{cM} 0$, according to the tumor-node-metastasis (TNM) classification system (Union for International Cancer Control, UICC 6th edition); iii) patient age of 20-80 years old; iv) Eastern Cooperative Oncology Group performance status of 0 or 1; v) no bowel obstruction; vi) no history of chemotherapy or radiotherapy; and vii) written informed consent given by the patient. The exclusion criteria were as noted below: i) Abscess formation surrounding the tumor; ii) a tumor with perforation which required an emergency operation. From May 2012 to December 2019, 67 consecutive patients were eligible to receive this therapy. Among them, 32 patients agreed to undergo this neoadjuvant therapy. The remaining 35 patients were treated with surgery alone (without neoadjuvant therapy) and were excluded from this study. The medical ethics committee of Saga Medical Center Koseikan reviewed and approved this study design (permission no. 19-11-01-03). 


\section{CRT protocol}

S-1 chemotherapy. S-1 was administrated orally twice daily on days 1-14 and 22-35. The dose of S-1 was assigned base on the body surface area (BSA). The initial dose of S-1 was determined as follows: BSA $<1.25,1.25-<1.5$ and $\geq 1.5 \mathrm{~m}^{2}$ received 80,100 and $120 \mathrm{mg} /$ day of $\mathrm{S}-1$, respectively.

Radiotherapy. Before irradiation, all patients underwent a simulation with computed tomography for 3-dimensional conformal radiotherapy. The clinical target volume included the primary tumor, mesorectal, internal iliac and presacral lymph nodes as well as the entire pelvic cavity. The levels of the superior and inferior border were placed at L5-S1 and the anal canal, respectively. The anterior and posterior borders were determined at the symphysis pubis and the sacrum, respectively. A total of 45 Gy of radiotherapy was started on the first day of administration of S-1 and delivered 5 times weekly with a daily fraction of 1.8 Gy for 5 weeks using a 3- or 4-field technique.

Surgery. Radical surgical resection was performed 4.1-10.6 weeks (median, 7.9 weeks) after the completion of CRT. Total or tumor-specific mesorectal excision along with the regional lymph nodes was performed. In addition, selective lateral lymph node dissection was performed in patients suspected of having lateral lymph node metastasis. All resected specimens were histopathologically analyzed. The pathologic TNM classification and stage were determined.

Evaluation of the short-term outcomes. The major toxicities, rate of treatment, response rate of neoadjuvant CRT, short-term clinical outcomes of neoadjuvant CRT, surgery-related complications, rate of curative resection and pathologic evaluation findings were assessed after treatment. The tumor shrinkage and clinical response were evaluated using the Response Evaluation Criteria in Solid Tumors (RECIST) v1.1. before surgery. Adverse events, including those of preoperative CRT and surgical complications, were evaluated after each treatment using Common Terminology Criteria for Adverse Events (CTCAE) v4.0. Pathological downstaging was defined as any reduction in the clinical $\mathrm{T}$ or $\mathrm{N}$.

Follow-up. The patients were followed up by undergoing blood tests, including tumor markers for CEA and CA19-9, every 3 months and chest to abdominal computed tomography every 6 months, until 5 years after the operation. Colonoscopy was done at 1,3 and 5 years after the operation.

\section{Results}

Patients. From May 2012 to December 2019, 32 patients were treated with this therapy. The patient and tumor characteristic are listed in Table I. The clinical tumor stage was cT3 in 24 patients $(75 \%)$ and cT4 in 8 patients $(25 \%)$. Nineteen patients (59\%) had clinical evidence of lymph node metastasis. Clinical stage II and III disease were noted in 13 (41\%) and 19 patients (59\%), respectively. The carcinoembryonic antigen (CEA) and carbohydrate antigen 19-9 (CA19-9) levels (tumor markers) before neoadjuvant therapy were $11.3 \pm 24.6 \mathrm{ng} / \mathrm{ml}$ and $19.3 \pm 16.1 \mathrm{U} / \mathrm{ml}$ (mean $\pm \mathrm{SD})$, respectively.
Table I. Patient and tumor characteristics $(n=32)$.

\begin{tabular}{lc}
\hline Characteristic & Value \\
\hline Sex, n (male:female) & $26: 6$ \\
Age, years (median; min, max) & $66.5(32,79)$ \\
Main tumor location, n (upper:lower rectum) & $4: 28$ \\
Histological differentiation, $\mathrm{n}($ well/mod:por/sig) & $28: 4$ \\
Clinical T classification, $\mathrm{n}(\mathrm{cT} / \mathrm{T} 4)$ & $24: 8$ \\
Clinical N classification, $\mathrm{n}(\mathrm{cN}-/+)$ & $13: 19$ \\
Clinical stage, $\mathrm{n}(\mathrm{cStage} \mathrm{II} / \mathrm{III})$ & $13: 19$ \\
Pretreatment & \\
Carcinoembryonic antigen, ng/ml (mean $\pm \mathrm{SD})$ & $11.3 \pm 24.6$ \\
Carbohydrate antigen 19-9, U/ml (mean $\pm \mathrm{SD})$ & $19.3 \pm 16.1$ \\
\hline
\end{tabular}

Acute adverse events associated with preoperative chemoradiotherapy. Table II shows the major toxicities observed among the patients according to the CTCAE. Anemia of all grades was observed in 24 patients $(75 \%)$ during the treatment. However, initially, 15 patients (47\%) experienced anemia. Anal pain (22 patients; 69\%) and skin and subcutaneous tissue disorders (19 patients; 59\%) were frequently seen in all grades. Grade $\geq 3$ leukopenia, anemia, neutrophil count reduction, platelet count reduction and diarrhea were seen in 2 patients (6\%), $1(3 \%), 1(3 \%), 1(3 \%)$ and 1 patient (3\%), respectively. Twenty-nine patients $(91 \%)$ completed this therapy without any change to the protocol or dosage. One patient experienced grade 2 malaise, and the dosage of the $\mathrm{S}-1$ was reduced. One patient postponed radiation therapy due to grade 2 diarrhea but completed the therapy after the interruption. One patient discontinued chemotherapy with S-1 on day 23 due to drug eruption.

Clinical tumor response. The clinical response is shown in Table III. Tumor shrinkage was seen in all patients, and the degree was $27.8 \pm 15.1 \%$ [mean \pm standard deviation (SD)] (Table III). A partial response and stable disease were seen in $12(38 \%)$ and 20 patients (63\%), respectively, according to the RECIST. Progressive disease was not seen in any patients. The CEA and CA19-9 levels before the operation were $3.8 \pm 3.9 \mathrm{ng} / \mathrm{ml}$ and $13.8 \pm 14.2 \mathrm{U} / \mathrm{ml}$ (mean $\pm \mathrm{SD}$ ), respectively.

Surgical treatment. Laparoscopic or open surgery was performed in $29(91 \%)$ and 3 (9\%) patients, respectively. The operative procedures were abdomioperineal resection (19 patients: 59\%), low anterior resection (6 patients: 19\%), intersphincteric resection (3 patients: $9 \%$ ), total pelvic exenteration (3 patients: 9\%) and Hartmann operation (1 patient: 3\%). Lateral lymph node dissection was performed in 5 patients (16\%). A diverting stoma with ileum was created in nine cases (28\%). All patients achieved R0 resection. No postoperative mortality was seen.

Postoperative complications. Postoperative complications were assessed according to the Clavien-Dindo classification. Abdominal abscess (grade II, 1 case; grade IIIa, 3 cases), delayed perineal wound healing (grade I, 3 cases; 
Table II. Acute adverse events associated with preoperative chemoradiotherapy.

Patients with adverse events of all

Adverse event grades, n $(\%)$
Patients with grade 3 and 4 adverse events, n (\%)

$\begin{array}{lrr}\text { Anemia } & 24(75) & 1(3) \\ \text { Leukopenia } & 10(31) & 2(6) \\ \text { Neutrophil count decreased } & 8(25) & 1(3) \\ \text { Platelet count decreased } & 11(34) & 1(3) \\ \text { AST and/or ALT increased } & 2(6) & 0(0) \\ \text { Anal pain } & 22(69) & 0(0) \\ \text { Skin and subcutaneous tissue disorders } & 19(59) & 0(0) \\ \text { Anorexia } & 8(25) & 0(0) \\ \text { Malaise } & 8(25) & 0(0) \\ \text { Skin hyperpigmentation } & 7(22) & 0(0) \\ \text { Nausea } & 6(19) & 0(0) \\ \text { Anal hemorrhage } & 4(13) & 0(0) \\ \text { Urticaria } & 3(9) & 0(0) \\ \text { Diarrhea } & 2(6) & 1(3)\end{array}$

AST, aspartate aminotransferase; ALT, alanine aminotransferase.

Table III. Clinical tumor response of patients $(n=32)$.

\begin{tabular}{lc}
\hline Finding & Value \\
\hline Tumor shrinkage, $\%($ mean $\pm \mathrm{SD})$ & $27.8 \pm 15.1$ \\
Clinical response, $\mathrm{n}(\%)$ & $0 \quad(0)$ \\
CR & $12(38)$ \\
PR & $20(63)$ \\
SD & $0 \quad(0)$ \\
PD & \\
Preoperation & $3.8 \pm 3.9$ \\
Carcinoembryonic antigen, $\mathrm{ng} / \mathrm{ml}($ mean $\pm \mathrm{SD})$ \\
Carbohydrate antigen $19-9, \mathrm{U} / \mathrm{ml}($ mean $\pm \mathrm{SD})$ & $13.8 \pm 14.2$ \\
\hline
\end{tabular}

CR, complete response; PR, partial response; $\mathrm{SD}$, stable disease; $\mathrm{PD}$, progressive disease.

grade IIIa, 1 case), bowel obstruction (grade II, 3 cases), pseudomembranous colitis (grade II, 2 cases), dysuria (grade I, 1 case; grade IIIa, 1 case), anastomotic leakage (grade IIIb, 1 case), anastomotic stenosis (grade IIIb, 1 case), urinary tract infection (grade II, 1 case), stenosis of the colostomy (grade II, 1 case) and cellulitis of the buttocks (grade II, 1 case) were seen. No postoperative mortality was observed.

Pathological evaluation. The pathological examination of the therapeutic effect was grade $0,1 \mathrm{a}, 1 \mathrm{~b}, 2$ and 3 in 0, 6, 22, 44 and $28 \%$ of cases, respectively (Table IV). Pathological complete response (pCR) was seen in 9 cases $(28.1 \%)$. Downstaging of tumor invasion and lymph node metastasis was observed in $14(44 \%)$ and $11(34 \%)$ cases, respectively. Combined $\mathrm{T}$ and/or $\mathrm{N}$ downstaging was seen in 19 cases (59\%).
Table IV. Pathologic results of patients $(n=32)$.

\begin{tabular}{lc}
\hline Finding & Value \\
\hline JSCCR pathological state, n (\%) & \\
Grade 0 & $0(0)$ \\
Grade 1a & $2(6)$ \\
Grade 1b & $7(22)$ \\
Grade 2 & $14(44)$ \\
Grade 3 & $9(28)$ \\
Pathological T classification, n (pT0/T2/T3/T4) & $10 / 3 / 18 / 1$ \\
Pathological N classification, n (pN-/+) & $21 / 11$ \\
Pathological stage, n (pCR/pStage I/II/III) & $9 / 3 / 9 / 11$ \\
T down-staging, n (\%) & $14(44)$ \\
N down-staging, n (\%) & $11(34)$ \\
Combined T and/or N down-staging, n (\%) & $19(59)$
\end{tabular}

JSCCR, Japanese Society for Cancer of the Colon and Rectum.

\section{Discussion}

Preoperative CRT is a key treatment for enhancing the local control and reducing the therapeutic toxicity compared with postoperative CRT. Preoperative CRT with continuous infusion of 5-FU or capecitabine is the standard treatment for rectal cancer $(1,13)$. 5-FU-based reagents function as radiosensitizers by killing S-phase cells, which are relatively radioresistant (14). Recently, capecitabine has been used to replace treatment with 5-FU as neoadjuvant chemoradiotherapy for locally advanced rectal cancer (13). However, a previous randomized study showed that CRT with capecitabine was associated with a significantly high incidence of hand-foot skin reaction (31\%; any grade), fatigue (28\%; any grade) and proctitis 
(16\%; any grade) compared with 5-FU (13). In contrast, S-1 is also used for adjuvant therapy after rectal surgery and is considered quite tolerable (15). The oral anticancer reagent $\mathrm{S}-1$ is a biochemical modulator of 5-FU and has been reported to enhance the effect of radiotherapy in cancer cells (7). Therefore, preoperative CRT with S-1 may be a viable candidate for the treatment of the rectal cancer.

In the present study, the frequent toxicity of this treatment was anal pain $(69 \%)$, followed by skin and subcutaneous tissue disorders (59\%). These toxicities are notable findings because no similar findings had ever been mentioned in previous reports $(10,11)$. However, it was a reversible event, and all patients recovered after the treatment before radical surgery. When performing chemoradiotherapy with S-1, we need to keep in mind the possible occurrence of skin related side effects. Regarding severe toxicity, grade 3/4 leukopenia, anemia, a neutrophil count reduction, a platelet count reduction and diarrhea were seen in 6, 3, 3, 3 and $3 \%$ of cases, respectively. These results were comparable to those of previous studies of preoperative CRT with 5-FU or capecitabine (leukopenia: 8.2 and $1.5 \%$, anemia: 1 and $0 \%$, platelet count reduction: 0.5 and 0 , diarrhea: 2 and $8.6 \%$, respectively) (13). One patient terminated treatment due to severe diarrhea. However, completion of this combined therapy without any modification was seen in $93.8 \%$ of cases of S-1 treatment and $96.9 \%$ of those with radiotherapy. Indeed, preoperative CRT with S-1 is reported to have a high compliance rate for chemotherapy $(89.2-100 \%)$ and radiotherapy (94.6-100\%) (10,11), with two reasons speculated: Oral treatment can be administrated on an outpatient basis, and S-1 is less toxic than intravenously delivered chemotherapeutic reagents (11).

A previous study showed that the $\mathrm{pCR}$ rate of CRT with S-1 was $10.8-22.2 \%(10,11)$. Our study showed a pCR rate of $28.1 \%$, which was consistent with the findings of previous reports. Capecitabine, an orally administrated pro-drug of 5 -FU, is already widely used in western countries, and the pCR rate of CRT with capecitabine reportedly ranges from $12-31 \%$ (16-18). In addition, the pCR rate of the floropyrimidine (5-FU, capecitabine, S-1)/irinotecan, floropyrimidine (5-FU, capecitabine)/oxaliplatin and floropyrimidine (5-FU, capecitabine)/bevacizmab with radiotherapy regimens for have been reported to be 13.7-37, 13.3-19.2 and 7.5-32\% and respectively (5). These results are comparable to those of CRT with S-1, and the tolerability of this therapy seems acceptable, suggesting that this regimen should be considered in the future.

In our study, R0 resection was performed in $100 \%$ of patients. A previous report on CRT with S-1 also found a similarly high R0 rate (94.6\%) (11). However, anal-preserving surgery was only performed in 9 patients $(28 \%)$ in our study, which seemed relatively low compared with the previous report of Hiratsuka et al (12) (43.2\%). Twenty-eight of 32 tumors were located in the lower rectum. Therefore, the anus could not be preserved in 19 patients with lower rectal cancer because a safe surgical margin could not be achieved due to the fact that the tumor was located close to the anus, and/or defecation dysfunction was deemed likely to occur due to the patient's age and background. On the other hand, among the 9 patients who achieved a pCR in whom the creation of a colostomy might have been avoidable, $6(67 \%)$ patients underwent the creation of a permanent colostomy. When performing radical surgery with the goal of achieving an R0 resection, it is sometimes not possible to successfully preserve the anus. In addition, our study included eight T4 cases and among them direct tumor invasion to either the bladder and/or prostate was diagnosed in 3 cases before performing neoadjuvant therapy. In these cases, computed tomography and/or magnetic resonance imaging before surgery still showed suspected direct invasion due to the unclear boundary with the surrounding organs. Thus, as a result, abdominoperineal resection was performed in all three cases. However, a pathological study demonstrated the depth of tumor invasion in those three cases to be pCR, T2 and T3, respectively. We therefore need to improve the diagnostic accuracy to avoid extended resection of the anus and the adjacent organs and to consider extending the indications for the preservation of the anal function and the adjacent organs on a case-by-case basis in the future.

The present study was associated with some limitations. First, the study was conducted at a single institution with a relatively small number of patients. In addition, we only examined the short-term results. Only one study from Hiratsuka et al (12) reported the long-term outcomes of CRT with S-1 for locally advanced rectal cancer, noting a 5-year disease-free survival and 5-year overall survival of 66.7 and $74.7 \%$, respectively, for Stage II and III rectal cancer. Therefore, further studies will be needed to confirm the efficacy of preoperative CRT with S-1 for locally advanced rectal cancer, including an assessment of the long-term outcomes regarding disease free survival and local recurrence, and sexual and urinary function.

In conclusion, we reported the efficacy of CRT with S-1. This therapy can be reasonably considered for locally advanced rectal cancer due to its acceptable toxicity and relatively high anti-tumor effect.

\section{Acknowledgements}

The authors would like to thank Dr Keiichiro Okuyama, Dr Hiroshi Kubo, Dr Osamu Ikeda, Dr Atsushi Miyoshi and Dr Seiji Sato who all belong to the Department of Surgery, Saga Medical Center Koseikan (Saga, Japan) for treating the patients and useful discussions. The authors would also like to thank Ms. Shiori Joujima (Medical Information Division, Life Science Research Institution, Saga Medical Center Koseikan, Saga, Japan) for data collection.

\section{Funding}

No funding was received.

\section{Availability of data and materials}

All data generated or analyzed during this study are included in this published article.

\section{Authors' contributions}

$\mathrm{MH}$, TW and TT contributed to the design of this study. $\mathrm{MH}, \mathrm{TT}$, HS and KK treated patients with chemotherapy and surgical procedure. YH, TW and HA treated patients with radiotherapy. MH, TT, YH, TW, HS, HA and KK contributed 
to acquisition of the clinical data. $\mathrm{MH}, \mathrm{YH}$ and $\mathrm{TT}$ analyzed the results. $\mathrm{MH}$ and TT drafted the manuscript. YH, TW, HS, $\mathrm{HA}$ and KK revised the manuscript. All authors read and approved the final manuscript.

\section{Ethics approval and consent to participate}

All patients and their families were fully informed about the chemoradiotherapy and surgical procedure in order to obtain their written consent, including broad consent to participate in the present study. The present study was reviewed and approved by the Saga Medical Center Koseikan Institutional Review Board (permission no. 19-11-01-03).

\section{Patient consent for publication}

Not applicable.

\section{Competing interests}

The authors declare that they have no competing interests.

\section{References}

1. Glynne-Jones R, Wyrwicz L, Tiret E, Brown G, Rödel C, Cervantes A and Arnold D; ESMO Guidelines Committee: Rectal cancer: ESMO clinical practice guidelines for diagnosis, treatment and follow-up. Ann Oncol 28 (Suppl 4): iv22-iv40, 2017.

2. Sauer R, Becker H, Hohenberger W, Rödel C, Wittekind C, Fietkau R, Martus P, Tschmelitsch J, Hager E, Hess CF, et al: Preoperative versus postoperative chemoradiotherapy for rectal cancer. N Engl J Med 351: 1731-1740, 2004.

3. Gérard JP, Conroy T, Bonnetain F, Bouché O, Chapet O, Closon-Dejardin MT, Untereiner M, Leduc B, Francois E, Maurel $\mathrm{J}$, et al: Preoperative radiotherapy with or without concurrent fluorouracil and leucovorin in T3-4 rectal cancers: Results of FFCD 9203. J Clin Oncol 24: 4620-4625, 2006.

4. Bosset JF, Collette L, Calais G, Mineur L, Maingon P, Radosevic-Jelic L, Daban A, Bardet E, Beny A and Ollier JC; EORTC Radiotherapy Group Trial 22921: Chemotherapy with preoperative radiotherapy in rectal cancer. N Engl J Med 355: 1114-1123, 2006.

5. Greenhalgh TA, Dearman C and Sharma RA: Combination of novel agents with radiotherapy to treat rectal cancer. Clin Oncol (R Coll Radiol) 28: 116-139, 2016.

6. Shirasaka T, Nakano K, Takechi T, Satake H, Uchida J, Fujioka A, Saito H, Okabe H, Oyama K, Takeda S, et al: Antitumor activity of $1 \mathrm{M}$ tegafur-0.4 M 5-chloro-2,4-dihydroxypyridine-1 M potassium oxonate (S-1) against human colon carcinoma orthotopically implanted into nude rats. Cancer Res 56: 2602-2606, 1996.
7. Zeng L, Ou G, Itasaka S, Harada H, Xie X, Shibuya K, Kizaka-Kondoh S, Morinibu A, Shinomiya K and Hiraoka M: TS-1 enhances the effect of radiotherapy by suppressing radiation-induced hypoxia-inducible factor-1 activation and inducing endothelial cell apoptosis. Cancer Sci 99: 2327-2335, 2008.

8. Takagi M, Sakata K, Someya M, Tauchi H, Iijima K, Matsumoto Y, Torigoe T, Takahashi A, Hareyama M and Fukushima M: Gimeracil sensitizes cells to radiation via inhibition of homologous recombination. Radiother Oncol 96: 259-266, 2010.

9. Sakata K, Someya M, Matsumoto Y, Tauchi H, Kai M, Toyota M, Takagi M, Hareyama $\mathbf{M}$ and Fukushima $\mathbf{M}$ : Gimeracil, an inhibitor of dihydropyrimidine dehydrogenase, inhibits the early step in homologous recombination. Cancer Sci 102: 1712-1716, 2011.

10. Sadahiro S, Suzuki T, Tanaka A, Okada K, Kamijo A, Murayama C, Akiba T and Nakayama Y: Phase I/II study of preoperative concurrent chemoradiotherapy with S-1 for locally advanced, resectable rectal adenocarcinoma. Oncology 81: 306-311, 2011.

11. Inomata M, Akagi T, Nakajima K, Etoh T, Tahara K, Matsumoto T, Ogawa T, Fujii K, Shiromizu A and Kitano S: A prospective feasibility study to evaluate neoadjuvant-synchronous S-1 with radiotherapy for locally advanced rectal cancer: A multicentre phase II trial. Mol Clin Oncol 4: 510-514, 2016.

12. Hiratsuka T, Etoh T, Hara T, Akagi T, Tahara K, Matsumoto T, Ogawa T, Fujii K, Shiromizu A, Shiroshita $\mathrm{H}$ and Inomata $\mathrm{M}$ : Long-term outcomes of neoadjuvant-synchronous S-1 plus radiotherapy for locally advanced rectal cancer: A multiinstitutional prospective phase II study. J Anus Rectum Colon 2: 168-175, 2018.

13. Hofheinz RD, Wenz F, Post S, Matzdorff A, Laechelt S, Hartmann JT, Müller L, Link H, Moehler M, Kettner E, et al: Chemoradiotherapy with capecitabine versus fluorouracil for locally advanced rectal cancer: A randomised, multicentre, non-inferiority, phase 3 trial. Lancet Oncol 13: 579-588, 2012.

14. Lawrence TS, Blackstock AW and McGinn C: The mechanism of action of radiosensitization of conventional chemotherapeutic agents. Semin Radiat Oncol 13: 13-21, 2003.

15. Hamaguchi T, Shimada Y, Mizusawa J, Kinugasa Y, Kanemitsu Y, Ohue M, Fujii S, Takiguchi N, Yatsuoka T, Takii Y, et al: Capecitabine versus $\mathrm{S}-1$ as adjuvant chemotherapy for patients with stage III colorectal cancer (JCOG0910): An open-label, non-inferiority, randomised, phase 3, multicentre trial. Lancet Gastroenterol Hepatol 3: 47-56, 2018.

16. Kim JS, Kim JS, Cho MJ, Song KS and Yoon WH: Preoperative chemoradiation using oral capecitabine in locally advanced rectal cancer. Int J Radiat Oncol Biol Phys 54: 403-408, 2002.

17. Kim JC, Kim TW, Kim JH, Yu CS, Kim HC, Chang HM, Ryu MH, Park JH, Ahn SD, Lee SW, et al: Preoperative concurrent radiotherapy with capecitabine before total mesorectal excision in locally advanced rectal cancer. Int J Radiat Oncol Biol Phys 63: 346-353, 2005.

18. Krishnan S, Janjan NA, Skibber JM, Rodriguez-Bigas MA, Wolff RA, Das P, Delclos ME, Chang GJ, Hoff PM, Eng C, et al: Phase II study of capecitabine (Xeloda) and concomitant boost radiotherapy in patients with locally advanced rectal cancer. Int $\mathbf{J}$ Radiat Oncol Biol Phys 66: 762-771, 2006. 